\title{
Numerical investigation of the electrical current effect on the fibrous tissue growth around biomaterials*
}

\author{
Shant Mahserejian ${ }^{1}$, Paul Ryan ${ }^{1}$, Rabia Djellouli ${ }^{2}$, and Randy Cohen ${ }^{3}$
}

\begin{abstract}
The objective of this study is to assess numerically the effect of applying electrical current on the fibrous tissue growth around polyethylene disk-shaped implants while subcutaneously placed inside 60 day old male Han-Wistar rats. This problem can be formulated as a design problem where the goal is to determine the parameters of a partial differential operator to achieve a desired effect. These electrical current parameters are computed using a regularized iterative method. The obtained results reveal that employing an appropriate electrical current profile can reduce the fibrous tissue concentration around the considered implant by up to $80 \%$. This preliminary study tends to demonstrate the effectiveness of this novel and non-invasive approach to shield the implant from intolerable levels of fibrous tissue growth, which consequently expands the lifespan and functionality of implanted devices, as well as avoids costly and traumatic surgical procedures.
\end{abstract}

\section{INTRODUCTION}

Implantation of biomaterials creates a foreign-body immune response. This includes the formation of a fibrous capsule to shield the body from the foreign object by creating a fibrous wall of tissue of varying thickness that surrounds or encapsulates the implant [1]-[2]. The formation of fibrous tissue around any implant is normal, and is an integral part of the wound-healing response to the implant. Problems begin to surface when the fibrous capsule squeezes the implant to the point where it hampers the performance of the implant, ultimately leading to its failure. Thus, invasive surgery to remove the fibrous formation or the implant is inevitable. Electrical current based techniques have emerged for addressing several challenging issues such as embryonic stem cell proliferation, electro-dessication and curettage for skin and liver cancer removal and treatment, burn wound healing by pulsed electrical stimulation, and radio-frequency ablation for inoperable liver cancer [3]-[4]. Hence, applying a moderate, non-harmful electrical current based approach appears to be a very attractive non-invasive alternative approach for disrupting the fibrous tissue growth around implanted biomaterials.

Given that, we propose to investigate numerically the feasibility and limitations of applying a moderate electrical current to prevent or diminish the effect of the fibrous tissue growth occurring around biomaterial implants. More

\footnotetext{
*This work is supported by MiniMed/Medtronic under Grant \# E1515

${ }^{1}$ S. Mahserejian and P. Ryan are with the Interdisciplinary Research Institute for the Sciences (IRIS), California State University Northridge (CSUN), USA smahsereand.edu, paul.ryan.201@my.csun.edu

${ }^{2} R$. Djellouli is with the Department of Mathematics \& IRIS, California State University Northridge (CSUN), USA rabia.djellouli@csun.edu

${ }^{3} \mathrm{R}$. Cohen is with the Department of Biology \& IRIS, CSUN, USA randy. cohendcsun. edu
}

specifically, our goal is to determine numerically the optimal electrical parameters, such as magnitude of the current, frequency, and duration of exposure, needed to disrupt the fibrous capsule tissue growth around a given implanted biomaterial. The determination of these parameters can be accomplished by solving an inverse problems that falls into the category of design problems consisting of estimating parameters of a partial differential operator to achieve a desired effect. The proposed study utilizes the mathematical model suggested [5] to describe the fibrous tissue growth around polyethylene disk-shaped implants subcutaneously placed inside 60-day old male Han-Wistar rats. The exposure of the subjects to the electricity is represented by a source term added to the considered model [5]-[6].

This numerical investigation demonstrates the effectiveness of using electricity for disrupting the fibrous tissue growth around implanted biomaterials. Indeed, the obtained results reveal that it is possible to design an admissible electrical current profile that can reduce the fibrous tissue density by up to $80 \%$, or if desired, confine it to a prescribed tolerable level. This preliminary study illustrates the great potential of this novel non-invasive approach for benefitting the biomedical community in several ways, chief among them extending the lifespan and functionality of implanted biomaterial devices, and avoiding the cost and trauma associated with repeated surgical procedures for dealing with the undesired fibrous tissue growth.

\section{PROBLEM STATEMENT}

The growth of fibrous tissue around homogeneous, discshaped implants exposed to electrical current can be modeled by the following initial boundary value problem $(I B V P)$ [5][6]:

$$
\begin{aligned}
& \begin{aligned}
\frac{\partial u}{\partial t}(r, t)= & c_{1} \frac{\partial}{\partial r}\left[\mathrm{e}^{-c_{2} r^{2}} \frac{\partial u}{\partial r}(r, t)\right]-c_{3} u(r, t) \\
& -K \sum_{n=0}^{N} k_{n} \chi_{[0, \omega]}(t-n p) u(r, t)
\end{aligned}
\end{aligned}
$$

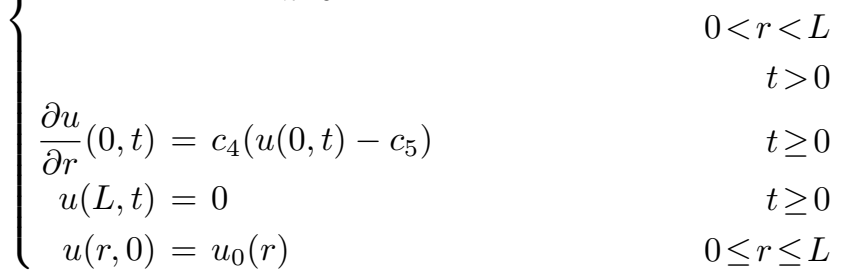

The concentration profile $u(r, t)$ is a function of the perpendicular distance away from the surface of the implant 
$r$, and the time, $t$. The first equation indicates that the capsule forming agents diffuse outward forming the capsule. The diffusion rate is affected by the sensitivity of the surrounding tissue to the implant and pressure it exerts. This is represented by the exponential factor $e^{-c_{2} r^{2}}$, which can be viewed as a cell-motility function. The natural removal of extraneous connective tissue is represented by the term $c_{3} u(r, t)$. The summation term in the first equation represents the electrical current present in the form of pulses. $k_{n}$ is the intensity (in $\mathrm{mA}$ ) of the $n^{\text {th }}$ pulse, $N$ is the total number of pulses, $p$ is the period between the pulses, $\omega$ is the pulse-width determining the exposure time $(\omega \leq p)$, and the total exposure time is $N p$. The proportionality constant $K$ is introduced to convert the voltage intensity to a nondimensional quantity. The value of $K$ was determined using in vivo data. Each individual square-shaped electrical current pulse is represented by the characteristic function $\chi_{[0, \omega]}$. Hereafter, we denote $\vec{k}=\left(k_{1}, k_{2}, \ldots, k_{N}\right)$. The Robin-type boundary condition characterizes the interaction between the implant and the capsule tissue. It indicates that the capsuleforming agents are deposited at the surface of the disc at a rate proportional to the difference between the concentration of tissue and the maximum level of concentration $c_{5} . t^{*}$ is the starting time of the electrical current, whereas $u_{0}(r)$ is the existing fibrous tissue concentration around the disc at $t^{*}$. Note that if $t^{*}=0$, then $u_{0}(r)=0 . c_{1}, c_{2}, c_{3}, c_{4}$, and $c_{5}$ are positive constants whose values depend on the physical and chemical properties of the implants as well as the characteristics of the test subjects. These values determine the nature of the capsule tissue growth: density, thickness, as well as deposition rate. Hence, these parameters are the biocompatibility index of the considered implant. In the considered case of low density polyethylene discs $(13 \mathrm{~mm}$ diameter and $2.5 \mathrm{~mm}$ thickness) implanted subcutaneously into 60 day old male Han-Wistar rats, the value of the biocompatible index $\vec{C}$ as well as the proportionally constant $K$ has been determined using in vivo measurements [6]. These values are reported in Table I.

Before concluding this section, we must point out that that $I B V P$ is different from the initial boundary value problem introduced in reference [5] by the summation term in the right-hand side, and a non homogeneous initial condition. In the absence of these two terms, $I B V P$ describes the averaged behavior of the concentration $u(r, t)$ of the fibrous tissue growth around an implanted disk.

TABLE I

BIOMATERIAL INDEX AND CONSTANT OF PROPORTIONALITY VALUES

\begin{tabular}{|c|c|c|c|c|c|}
\hline$c_{1}$ & $c_{2}$ & $c_{3}$ & $c_{4}$ & $c_{5}$ & $K$ \\
\hline 18.9 & 0.00009 & 0.000042 & 6.57 & 18.5 & 0.007 \\
\hline
\end{tabular}

\section{THE DESIGN PROBLEM}

The goal is to find the optimal values of period, pulsewidth, and intensity which will minimize the tissue concentration around the considered disc-shaped implant. To this end, we first observe that the solution to $I B V P$ defines an operator $\mathbf{F}$ that maps the electrical profile parameters $\vec{k}=\left(k_{1}, k_{2}, \ldots, k_{N}\right), \omega$, and $p$ to the concentration profile $u(r, t)$. The determination of these parameters, which reduce the fibrous capsule tissue concentration $u$ for a prescribed duration of electricity exposure $N p$, can be formulated as the following minimization problem:

Given an initial measured fibrous concentration $u_{0}(r)$, find an admissible electrical current profile $\left(\overrightarrow{k^{*}}, \omega^{*}, p^{*}\right)$ such that:

$$
\left(\overrightarrow{k^{*}}, \omega^{*}, p^{*}\right)=\operatorname{argmin}\|\mathbf{F}(\vec{k}, \omega, p)-\tilde{u}\|_{2}
$$

where $\tilde{u}$ is a prescribed tolerable concentration level of the fibrous tissue, and $\|\cdot\|_{2}$ is the Euclidian norm. Observe that if $\tilde{u} \equiv 0$, then the minimization problem (1) consists of finding $\left(\overrightarrow{k^{*}}, \omega^{*}, p^{*}\right)$ that will bring down the concentration of the capsule tissue to its minimum possible level, whereas if $\|\tilde{u}\|_{2} \neq 0$, then the obtained electrical current will prevent the fibrous tissue growth from exceeding the prescribed concentration level $\tilde{u}$.

\section{SOLUTION METHODOLOGY}

As stated earlier, the minimization problem (1) is an inverse problem that falls into the category of design problems. This problem is nonlinear and ill-posed in the sense of Hadamard [7] which make it difficult to solve. In order to overcome these difficulties, we use a regularized Newtontype method. The iterative Newton method addresses the nonlinear aspect of the problem, while the Tikhonov regularization procedure addresses its ill-posed nature. Next, we briefly highlight the salient features of the proposed solution methodology.

We set $\vec{p}=\left(k_{1}, k_{2}, \ldots, k_{N}, \omega, p\right)^{T}$, then solving the nonlinear problem (1) by the classical Newton method requires, at each iteration $l$, that we solve the following linear system:

$$
\mathbf{J}_{F}^{l} \delta \overrightarrow{\boldsymbol{p}}^{l}=\tilde{\mathbf{u}}-\mathbf{F}\left(\boldsymbol{\rho}^{l}\right)
$$

where $\mathbf{J}_{F}$ is the Jacobian matrix corresponding to the operator $\mathbf{F}$. Then, we update:

$$
\overrightarrow{\boldsymbol{p}}^{l+1}=\overrightarrow{\boldsymbol{p}}^{l}+\delta \overrightarrow{\boldsymbol{p}}^{l} \text {. }
$$

Since the number of concentration measurements $u$ is typically greater than the number of the sought-after electrical current parameters $\vec{p}$, the resulting linear system (2) is overdetermined. Therefore, we solve it in the least-squares approximation, as follows:

$$
\mathbf{J}_{F}^{l}{ }^{T} \mathbf{J}_{F}^{l} \delta \overrightarrow{\boldsymbol{p}}^{l}=\mathbf{J}_{F}^{l}{ }^{T}\left(\tilde{\mathbf{u}}-\mathbf{F}\left(\overrightarrow{\boldsymbol{p}}^{l}\right)\right)
$$

where $\mathbf{J}_{F}^{l}{ }^{T}$ is the transpose of the Jacobian matrix of the operator $\mathbf{F}$ at iteration $l$. Note that the linear system (4) is illconditioned due to the ill-posed nature of the inverse problem (1). For this reason, we employ a Tikhonov regularization procedure to stabilize it. Consequently, the linear system (4) is replaced by:

$$
\left(\mathbf{J}_{F}^{l}{ }^{T} \mathbf{J}_{F}^{l}+\mu I\right) \delta \overrightarrow{\boldsymbol{p}}^{l}=\mathbf{J}_{F}^{l}{ }^{T}\left(\tilde{\mathbf{u}}-\mathbf{F}\left(\overrightarrow{\boldsymbol{p}}^{l}\right)\right)
$$


where $I$ is the identity matrix and $\mu$ is a positive number, called the regularization parameter. Note that larger values of $\mu$ improve the condition number of the linear system (5), whereas smaller values of $\mu$ lead to a greater accuracy. Therefore, the convergence as well as the accuracy of the regularized Newton algorithm depends strongly on the choice of the value of $\mu$. The "optimal" $\mu$ is found here through a trial and error strategy consisting of sweeping the values of $\mu$ in a geometric progression while measuring the corresponding relative residual.

The critical step in the numerical implementation of the proposed regularized Newton method is the computation, at each iteration $l$, of the Jacobian matrix $\mathbf{J}_{F}$. Such computation must be executed with a high level of accuracy to ensure the stability, fast convergence, and computational efficiency of the proposed algorithm. We have established in [6] that the derivative of $\mathbf{u}$ with respect to parameter $\rho_{j}$ (the $j^{\text {th }}$ coordinate of $\vec{p}$ ) are the solutions of $I B V P$ with different righthand sides. Consequently, at each iteration $l$, the computation of the Jacobian matrix $\mathbf{J}_{F}^{l}$ requires solving the same initial boundary value problem with $N+3$ different right hand sides, $N$ being the total number of pulses (See Eq. (1)).

We employed a Crank-Nicolson type finite difference scheme to solve the direct problems of type $I B V P$ that incur at each iteration. More specifically, we subdivided the interval $[0, L]$ uniformly in $J$ subsets $\left[r_{j}, r_{j+1}\right]$; for $j=0, \ldots, J$, where $r_{j}=j \Delta x$ (measured in $\mu \mathrm{m}$ ). Similarly, we considered for the time variable $t$, the discrete set $t^{m}=m \Delta t ;(\Delta t$ measured in seconds/milliseconds). We then approximated the firstand second-order derivatives in space in $(I B V P)$ using the following fourth-order approximations in space [8]:

$$
\begin{gathered}
\frac{\partial u}{\partial r}\left(r_{j}, t^{m}\right) \approx \frac{u_{j-2}^{m}-8 u_{j-1}^{m}+8 u_{j+1}^{m}-u_{j+2}^{m}}{12 \Delta r} \\
\frac{\partial^{2} u}{\partial r^{2}}\left(r_{j}, t^{m}\right) \approx \frac{-u_{j-2}^{m}+16 u_{j-1}^{m}-30 u_{j}^{m}+16 u_{j+1}^{m}-u_{j+2}^{m}}{12 \Delta r^{2}}
\end{gathered}
$$

Moreover, the first-order derivative in time was approximated using the following second-order scheme [8]:

$$
\frac{1}{2}\left[\frac{\partial u}{\partial t}\left(r_{j}, t^{m+1}\right)+\frac{\partial u}{\partial t}\left(r_{j}, t^{m}\right)\right] \approx \frac{u_{j}^{m+1}-u_{j}^{m}}{\Delta t}
$$

Observe that computing $u_{j}^{m}$ requires the knowledge of the two neighboring points both to the left and right, as shown in Fig. 1. Therefore, the construction of the boundary points $u_{0}^{m}$ and $u_{1}^{m}$ requires the computation of fictitious concentration values. These are estimated using the following approximations:

$$
\begin{aligned}
& u\left(r_{-2}, t\right) \approx u_{2}^{m}-4 \Delta r c_{4}\left(u_{0}^{m}-c_{5}\right) \\
& u\left(r_{-1}, t\right) \approx-\frac{3}{2} u_{0}^{m}+3 u_{1}^{m}-\frac{1}{2}-3 \Delta r c_{4}\left(u_{0}^{m}-c_{5}\right)
\end{aligned}
$$

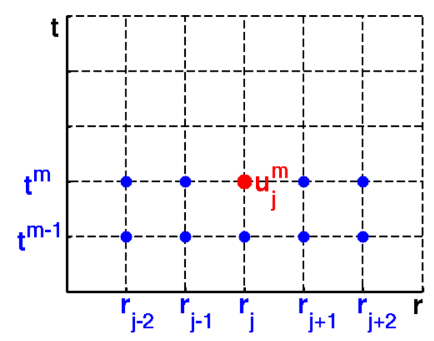

Fig. 1. A schematic interpretation of spatial dependence for the considered finite difference discretization.
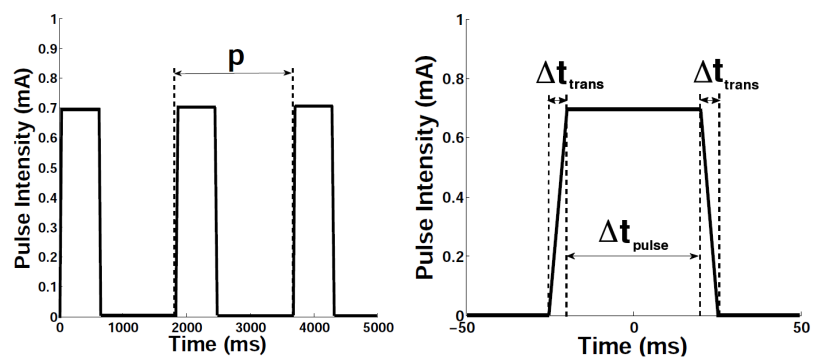

Fig. 2. Time scale discretization. The periodic separation between electric pulses (left), the time steps within each pulse (right)

For the opposite end of the spatial spectrum, $j \geq J$, since no tissue growth occurs, we set $u_{J}^{m}=u_{J+1}^{m}=0$.

The approximation of the electrical source terms deserves a special attention. This is due to the fact that the period between pulses $p$, is measured in seconds, whereas the pulse-width $\omega$ is measured in milliseconds. Moreover, the discontinuity of the characteristic function $\chi_{[0, w]}$ calls for a very small time step to transition in and out of the current pulses in order to maintain the effect of the electrical presence numerically. Together, these concerns prompted the need for a multi-step scheme in time, as illustrated in Fig. 2. We set $\Delta t_{s}=1$ second. Then, we set $\Delta t_{\text {trans }}=10^{-3} \Delta t_{s}$ for transitioning in/out of pulses, and we set $\Delta t_{\text {pulse }}=$ $\omega 10^{-3} \Delta t_{s}\left(\right.$ resp. $\left.\Delta t_{\text {pulse }}=\Delta t_{s}\left(p-10^{-3}(\omega+2)\right)\right)$ while the electrical pulses were on (resp. off).

Application of the proposed finite difference scheme leads to these linear systems:

$$
A^{m+1} u^{m+1}=b^{m} ; \quad m \geq 0
$$

where $A^{m+1}$ is a $(J \times J)$ non-symmetric matrix with five nonzero diagonals, $u^{m+1}$ is the vector $\left(u_{0}^{m+1}, u_{1}^{m+1}, \ldots, u_{J-1}^{m+1}\right)^{T}$ consisting of concentration profile values at each point $r_{j}$ and at time $t^{m+1}$, and $b^{m}$ is a vector whose components are a linear combination of the components of the vector $u^{m}$, the concentration profile at the previous time step $m$. Since $A^{m+1}$ is sparse and relatively small (typically less than $1000 \times 1000$ ), we employ the classical LU-factorization method for solving the resulting linear systems (9). 

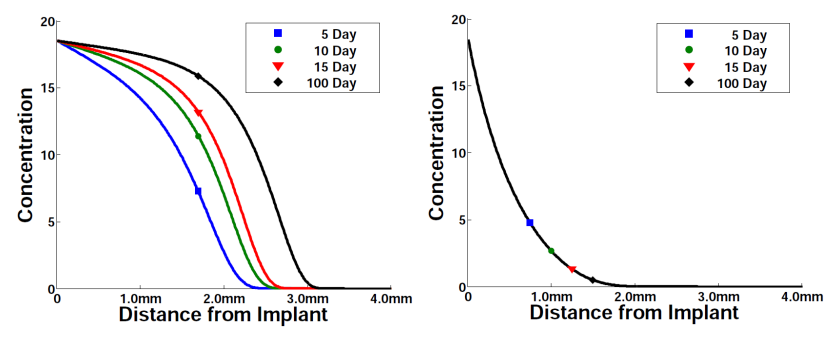

Fig. 3. Tissue growth concentration at various times. Fibrous capsule tissue growth in the absence of electricity (left), and fibrous capsule tissue growth exposed to a continuous electrical exposure (right).

\section{NUMERICAL EXPERIMENTS}

We analyze in this section the effect of the electrical current by performing the following two sets of experiments.

\section{Experiment \#1: Effect of the electrical current when applied at a prescribed time.}

In this numerical experiment, a continuous electrical current is applied at the implantation time, that is, at time $t^{*}=0$. Hence, the initial concentration profile in $I B V P$ is $u_{0}(r)=$ $0 ; \forall r \in[0, L]$. The computed admissible optimal current intensity in this case delivered by the Newton algorithm is $\mathbf{k}^{*}=1.0 \mathrm{~mA}$. We report in Fig. 3 (left) the growth of the concentration profile in the absence of any current after 5, 10,15 , and 100 days, as well as the growth when subject to the electrical current delivered by the Newton algorithm (see Fig. 3 (right)). The results indicate the following:

- In the absence of electrical current, the concentration profile grew consistently until it attained a maximum level (asymptotic regime) after 100 days.

- When applying the electrical current, the growth of the capsule tissue also reached a maximum growth level. However, the total cellular growth level was approximately $80 \%$ less than the maximum growth in the absence of electrical current. Clearly, the application of a continuous electrical current significantly disrupted the growth of the tissue around the considered implant.

\section{Experiment \#2: Electrical current applied periodically.}

In the following experiment, we apply the electrical current periodically to confine the growth of the fibrous tissue concentration below a tolerable level. We assume here that the maximum growth level that can be tolerated is $50 \%$ of the maximum growth observed (see Fig. 3). Hence, beginning at time $t^{*}$ (at which the concentration level reached 50\% of the maximum possible growth), we applied the electrical current periodically for one day on and two consecutive days off. The results depicted in Fig. 4, demonstrate that it is possible to confine the tissue concentration at a prescribed level (between 25\% and 50\% in this case) with a single day's dose of electrical current. This experiment suggests that, even if it isn't possible to completely disrupt or remove the fibrous tissue, a periodic repellent dose controls and confines the tissue growth to a reduced and tolerable level.

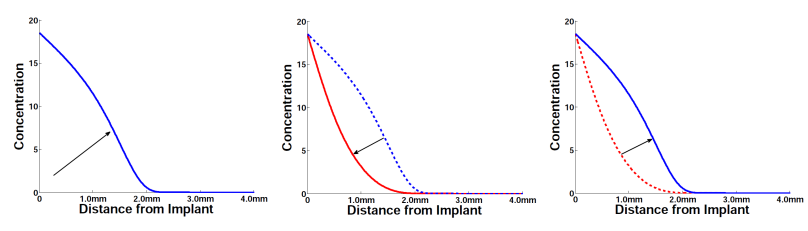

Fig. 4. Periodic application of continuous electrical current profile beginning with admissible initial growth for 2.5 days (left), electricity exposure for 1 day (center), and admissible growth for 2 days (right).

\section{CONCLUSIONS}

We have investigated numerically the feasibility and limitations of applying an electrical current-based procedure for disrupting fibrous capsule tissue formation around biomaterial implants. This study reveals that applying a moderate electrical current allows a significant reduction and/or confinement at a tolerable level of the concentration of the fibrous tissue surrounding the implant. This preliminary study provides initial and practical guidelines for applying the electrical current, rather than tedious trial and error to determine suitable electrical repulsion of encapsulation. This in turn has the potential to greatly reduce the duration and costs of in vivo experiments that need to be performed for verification and validation purposes. We are currently conducting these experiments by implanting subcutaneously disk-shaped implants in rats. This study is in compliance with current approval of CSUNs IACUC committee.

\section{ACKNOWLEDGMENT}

The authors would like to thank Dr. Marie Tieck and Ms. Lavotia Dermendjian for the enlightening discussions. Finally, they thank Mary Komazdjian, Michael Silva, and Shahab Younesi for their assistance with the in vivo laboratory experiments. Any opinions, findings, conclusions, or recommendations expressed in this material are those of the authors and do not necessarily reflect the views of MiniMed/Medtronic or CSUN.

\section{REFERENCES}

[1] D. J. Li, K. Ohsaki, K. Li, P. C. Cui, Q. Ye, K. Baba, Q. C. Wang, S. Tenshin, and T. T. Yamamoto, "Thickness of fibrous capsule after implantation of hydroxyapatite in subcutaneous tissue in rats", $J$. of Biomedical Materials Research, vol. 45, pp. 322-326, 1999.

[2] R. Kontio, P. Ruuttila, L. Lindroos, R. Suuronen, A. Salo, C. Lindqvist, I. Virtanen, and Y. T. Konttinen, "Biodegradable polydioxanone and poly(L/D)lactide implants: an experimental study on peri-implant tissue response", J. Oral Maxillofac. Surg., vol. 34, pp. 766-776, 2005.

[3] M. M. Sackenheim, " Radio Frequency Ablation: The Key to Cancer Treatment", J. of Diagnostic Medical Sonography, vol. 19(2), pp. 8892, 2003.

[4] A. W. Kopf, R. S. Bart, D. Schrager, M. Lazar, G. L. Popkin, "Curettage-electrodessication treatment of basal cell cacinomas", Archives of Dermatology, vol. 113(4) pp. 439-43, 1997.

[5] P. Ryan, R. Djellouli, and R. Cohen, "Modeling capsule tissue around disk-shaped implants: a numerical and in vivo study", J. Mathematical Biology, vol. 57, pp. 675-695, 2008.

[6] S. Mahserejian, "Electrical Based Therapy to Treat the Fibrous Capsule Tissue Growth Occurring Around Subcutaneous Implants" California State University Northridge Masters Thesis, 2012.

[7] J. Hadamard, "Lectures on Cauchy's Problem in Linear Partial Differential Equations", Yale University Press, New Haven, 1923.

[8] J.C. Strikwerda, "Finite difference schemes and partial differential equations, 2d ed." SIAM, 2004. 